\title{
Intermittency of earthquake cycles in a model of a three-degree-of-freedom spring-block system
}

\author{
Y. Abe $^{1}$ and N. Kato ${ }^{2}$ \\ ${ }^{1}$ ITOCHU Techno-Solutions Corporation, Tokyo, Japan \\ ${ }^{2}$ Earthquake Research Institute, University of Tokyo, Tokyo, Japan \\ Correspondence to: Y. Abe (yuta.abe.150@ctc-g.co.jp)
}

Received: 12 November 2013 - Revised: 16 May 2014 - Accepted: 19 May 2014 - Published: 11 August 2014

\begin{abstract}
We herein report the results of some numerical simulations of complex earthquake cycles using a threedegree-of-freedom spring-block model with a rate- and statedependent friction law. The model consists of three blocks on a conveyor belt that is moving at a steady rate. Observed complex slip behaviour in the simulations is classified into five slip patterns, and for each of these the parameter dependence of the slip patterns is demonstrated by means of phase diagrams. Aperiodic slip patterns occur for wider ranges of the parameter space in the three-block system than in the two-block system. Chaotic slip behaviour known here as "intermittency" is found in the three-block system, in which two different slip patterns occur alternately with variable durations. By calculating Lyapunov exponents, we quantify the dependence of slip evolution on the initial conditions for each slip pattern. For cases where intermittent slip patterns occur, the time evolution of the Lyapunov exponent is correlated with changes in slip behaviour.
\end{abstract}

\section{Introduction}

The accurate forecasting of earthquakes requires an understanding of the complexity of patterns of earthquake occurrence; in particular, the interaction between fault segments is one of the key factors that determine the complexity of an earthquake cycle. A two-degree-of-freedom spring-block model, which consists of two rigid blocks connected by an elastic spring and loaded at a constant rate, is the simplest model of stick-slip and earthquake cycles and is used to study the effects of fault interaction. Nussbaum and Ruina (1987) conducted simulations using the two-block system with constant static and dynamic friction, and suggested that a complex earthquake pattern may occur when the properties of the material are uniform. Using the two-block system with velocity-weakening friction and the appropriate model parameters, Huang and Turcotte (1990) successfully simulated earthquake cycles similar to those found along the southcentral San Andreas Fault, California, and in the Nankai Trough, southwestern Japan. Through a systematic examination of the same model, Huang and Turcotte (1992) found a transition in slip behaviour from periodic to chaotic through repeated period-doubling bifurcations.

Various aseismic slip events have recently been observed (Schwartz and Rokosky, 2007). These events cause perturbances to the stresses, possibly affecting earthquake occurrence. However, aseismic slip cannot be reproduced by simple friction in terms of constant static and dynamic or velocity-weakening friction. In contrast, rate- and statedependent friction laws (Dieterich, 1979; Ruina, 1983) can be used to simulate both seismic and aseismic sliding. Using a two-degree-of-freedom spring-block model with a rate- and state-dependent friction law, Ma and He (2001) examined complex sliding processes and found that period-doubling bifurcation occurred for some friction parameters, in which large events and small events occurred alternately. Using a similar two-block system, Yoshida and Kato (2003) examined the interactions between a block with unstable frictional properties and a block with stable or conditionally stable frictional properties in order to explain the occurrence of slow earthquakes.

Abe and Kato (2012; hereafter Paper 1) conducted a systematic parameter study using a two-degree-of-freedom spring-block model assuming a rate- and state-dependent friction law, and produced phase diagrams of slip patterns including the periodic recurrence of seismic and aseismic slip 
events and aperiodic earthquake cycles. Aseismic slip events occur when the spring stiffness is close to the critical value for the occurrence of unstable slip. Aperiodic slip patterns resulting from interactions were not observed when the stiffness of the coupling spring between the two blocks was relatively weak. When both seismic and aseismic slip events occurred at a block, aseismic slip events were found to complicate the recurrence pattern of earthquakes in some cases.

The two-degree-of-freedom spring-block models may be too simplistic to allow the reproduction of observed seismicity, and complexity in synthetic seismicity is expected to increase with the number of degrees of freedom. Nomanbhoy and Ruff (1996) used a spring-block system consisting of three blocks, in which aseismic sliding is assumed to occur at one of the blocks, to simulate complex earthquake sequences including earthquake doublets. Mitsui and Hirahara (2004) used a model consisting of five blocks with rate- and statedependent friction to simulate earthquake sequences along the Nankai Trough in southwestern Japan. Although these studies succeeded in simulating earthquake sequences similar to the observed ones, they did not conduct systematic studies of parameters nor did they examine the statistical properties of simulated earthquakes. Moreover, the difference in the complexity of the synthetic seismicity between the two-block and larger systems may be particularly interesting. Erickson et al. (2011) investigated the slip behaviour of a multi-block system with rate- and state-dependent friction and found that chaotic slip patterns occur in some cases. The slip velocities simulated in Erickson et al. (2011) were much smaller than seismic slip velocities $\left(\sim 1 \mathrm{~m} \mathrm{~s}^{-1}\right)$. Slip behaviour at high slip rates must be properly taken into consideration in order to understand the complexity of earthquake sequences.

In this study, we conduct numerical simulations using a three-degree-of-freedom spring-block model with a rate- and state-dependent friction law. We examine the slip behaviour of the three-block system and compare it with the results obtained from the two-block system described in Paper 1. In particular, we focus on complex slip patterns that are not observed in the two-block system, and give statistical and dynamical analyses of these. The parameter dependence of slip behaviour is organized using phase diagrams for the periodicity of slip events through a wide and systematic parametric study. The parameter ranges where complex slip patterns or aseismic slip events are observed are expected to change with the increase in the number of degrees of freedom from two to three.

While the patterns of earthquake occurrence are statistically stationary in the two-block model (Paper 1), we find that the behaviour of the three-block system spontaneously switches between two earthquake occurrence patterns with random durations. This behaviour is known as "intermittency". Pomeau and Manneville (1980) used the Lorenz model, which is a simple dynamical system that exhibits complex behaviour, and found three types of inter- mittency. Several types of intermittency have been reported in numerical simulations of earthquake cycles. Ben-Zion et al. (1999) examined two different continuum fault systems and showed that each alternately exhibits two types of earthquake occurrence pattern (Lyakhovsky et al., 1999; Dahmen et al., 1998). The first is a pattern of clustering of large earthquakes with fewer intermediate-size earthquakes; the frequency-magnitude relationship of the large earthquakes is compatible with that of characteristic earthquake models (e.g. Wesnousky, 1994). The second is a pattern that includes earthquakes of various magnitudes, in which the frequencymagnitude relationship obeys a power law compatible with the Gutenberg-Richter relationship. Sándor et al. (2013) investigated the dynamics of a spring-block model proposed by Burridge and Knopoff (1967) and observed intermittency in both the experiments and computer simulations. Using a five-block system with a rate- and state-dependent friction law, Mitsui and Hirahara (2004) also found that the simulated earthquake occurrence pattern can spontaneously vary even under steady loading. Intermittent behaviour is important in earthquake occurrence patterns because it affects long-term forecasting of earthquakes using the statistical properties of earthquake recurrence. Geological studies suggest that large earthquakes on a fault are clustered within a short space of time (Weldon et al., 2004), implying that intermittency exists in natural earthquake sequences. Here, we analyse two types of slip pattern with intermittency observed in the present three-block system by examining the iteration maps for the recurrence interval, the time evolution of the Lyapunov exponents, and the probability distributions of the recurrence interval.

\section{Model}

In our three-degree-of-freedom spring-block model, we consider three rigid blocks on a conveyor belt that is moving at a speed of $V_{\mathrm{pl}}$ in the $x$ direction (Fig. 1). The blocks are connected by springs of stiffness $k_{12}$ and $k_{23}$ between Blocks 1 and 2 and Blocks 2 and 3, respectively, and each block is connected to a fixed wall by a spring of stiffness $k_{0}$. The equations of motion are written as

$$
\begin{aligned}
& m_{1} \mathrm{~d}^{2} x_{1} / \mathrm{d} t^{2}=-k_{0} x_{1}+k_{12}\left(x_{2}-x_{1}\right)-F_{\mathrm{n}} \mu_{1}, \\
& m_{2} \mathrm{~d}^{2} x_{2} / \mathrm{d} t^{2}=-k_{0} x_{2}+k_{12}\left(x_{1}-x_{2}\right)+k_{23}\left(x_{3}-x_{2}\right)-F_{\mathrm{n}} \mu_{2}, \\
& m_{3} \mathrm{~d}^{2} x_{3} / \mathrm{d} t^{2}=-k_{0} x_{3}+k_{23}\left(x_{2}-x_{3}\right)-F_{\mathrm{n}} \mu_{3},
\end{aligned}
$$

where $m_{i}, x_{i}$, and $\mu_{i}(i=1,2,3)$ are the mass, the position coordinate from the each reference point, and the coefficient of friction of the $i$ th block, respectively. We note that if the $i$ th block is stuck on the conveyor belt, it follows that $\mathrm{d} x_{i} / \mathrm{d} t=V_{\mathrm{pl}}$. The same normal force $F_{\mathrm{n}}$ is applied to each block.

The frictional stress at the base of each block is assumed to obey a rate- and state-dependent friction law (Dieterich, 


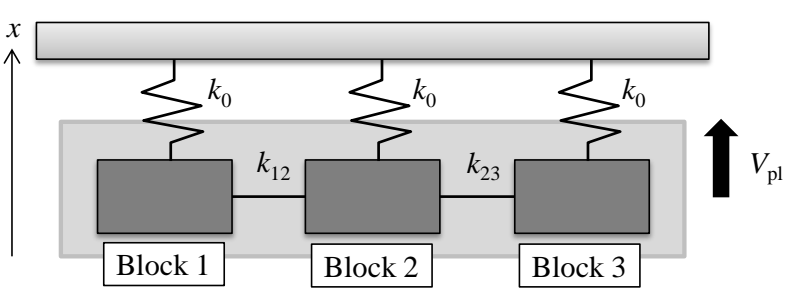

Figure 1. Schematic diagram of a three-degree-of-freedom springblock model. Blocks 1, 2, and 3 are connected to a fixed wall with springs of stiffness $k_{0}$, and connected to each other with springs of stiffness $k_{12}$ and $k_{23}$ between Blocks 1 and 2 and Blocks 2 and 3, respectively. The three blocks are driven by a belt conveyor moving at a rate of $V_{\mathrm{pl}}$.

1979; Ruina, 1983). The friction coefficient $\mu_{i}$ at the $i$ th block is given by

$\mu_{i}=\mu_{*}+a_{i} \ln \left(V_{i} / V_{*}\right)+b_{i} \ln \left(\theta_{i} / \theta_{*}\right)$,

$\mathrm{d} \theta_{i} / \mathrm{d} t=1-V_{i} \theta_{i} / L_{i}$,

where $V\left(=V_{\mathrm{pl}}-\mathrm{d} x / \mathrm{d} t\right)$ is the velocity relative to the conveyor belt, $\theta$ is a state variable, $L$ is a characteristic slip distance, and $a$ and $b$ are constants that represent the rate and time dependence of friction, respectively. $\mu_{*}$ and $\theta_{*}$ are the steady state values at a reference velocity $V_{*}$, which is chosen as $V_{\mathrm{pl}}$ in the present study. We use the "ageing" type of state evolution law for the differential equation of $\theta(2 b)$. We integrate Eqs. (1), (2a), and (2b) using a fifth-order Runge-Kutta method with adaptive time step control (Press et al., 1992).

In a single-degree-of-freedom spring-block model with spring stiffness $k$, stick-slip motion occurs for $a-b<0$ and $k<k_{\mathrm{c}}$ (Ruina, 1983), where the critical stiffness $k_{\mathrm{c}}$ is defined as

$k_{\mathrm{c}}=\frac{(b-a) F_{\mathrm{n}}}{L}$.

When $a-b>0$, the friction shows steady-state velocity strengthening, leading to stable sliding. Because we are interested in the interaction between the oscillating blocks, we set $a-b<0$ for the three blocks in this study. When some blocks are connected with springs, the slip motion of a block is controlled not only by the spring between the block and the driver but also by the springs connected to the neighbouring blocks, as discussed by Yoshida and Kato (2003) and in Paper 1. For example, when Block 1 is locked, it is dragged by Block 2 and the driver; this is equivalent to Block 1 being dragged by a spring of stiffness $k_{0}+k_{12}$. When $k_{0}>k_{\mathrm{ci}}$, where $k_{\mathrm{ci}}$ is the critical stiffness of the $i$ th block, stable slip is expected to occur at the $i$ th block, whether or not the other block is locked. Both stable and unstable slip could occur for $k_{0}<k_{\mathrm{ci}}<k_{0}+k_{12}$ as found in the simulation described in Paper 1. For $k_{0}+k_{12}<k_{\mathrm{ci}}$, unstable slip usually occurs. When the interaction with the neighbouring block is strong, creep-like behaviour may sometimes occur even for $k_{0}+k_{12}<k_{\mathrm{ci}}$ as shown in Paper 1 .
As discussed above, $k_{12} / k_{0}, k_{23} / k_{0},\left(k_{0}+k_{12}\right) / k_{\mathrm{c} 1},\left(k_{0}+\right.$ $\left.k_{12}+k_{23}\right) / k_{\mathrm{c} 2}$, and $\left(k_{0}+k_{23}\right) / k_{\mathrm{c} 3}$ may be regarded as control parameters in the present three-block system. In our numerical simulation, we assume $k_{12}=k_{23}$ for simplicity and define $K \equiv k_{12} / k_{0}, P_{1} \equiv\left(k_{0}+k_{12}\right) / k_{\mathrm{c} 1}, P_{2} \equiv\left(k_{0}+k_{12}+k_{23}\right) / k_{c 2}$, and $P_{3} \equiv\left(k_{0}+k_{23}\right) / k_{c 13}$. We fix the values of the loading spring stiffness $k_{0}$, the normal force $F_{\mathrm{n}}$, the masses of blocks $m_{1}, m_{2}$, and $m_{3}$, and the frictional parameters $a$ and $b$, while varying the coupling spring stiffness $k_{12}$ and the characteristic slip distance $L$. We assume $L_{1}>L_{3}$, and consequently $k_{\mathrm{c} 1}<k_{\mathrm{c} 3}$, which indicates that the slip motion of Block 3 is always less stable. The fixed values in the simulations presented herein are as follows: $F_{\mathrm{n}}=5.0 \times$ $10^{18} \mathrm{~N}, k_{0}=1.0 \times 10^{16} \mathrm{~N} \mathrm{~m}^{-1}, a_{1}=a_{2}=a_{3}=1.0 \times 10^{-3}$, $b_{1}=b_{2}=b_{3}=1.2 \times 10^{-3}, m_{1}=m_{2}=m_{3}=6.0 \times 10^{17} \mathrm{~kg}$, and $V_{\mathrm{pl}}=4.0 \mathrm{~cm}^{\text {years }}{ }^{-1}$. These values are the same as those assumed in Paper 1, where the parameters were set such that the simulated slip would be similar to those of the earthquake cycles along the Nankai Trough, following Mitsui and Hirahara (2004).

We conducted simulations systematically for $K=0.2$ and 1.0 ; in Paper 1 we used a similar systematic parameter study using $K=0.05,0.2$, and 1.0. We did not apply $K=0.05$ here because no chaotic slip pattern was observed in the two-block system for $K=0.05$, as reported in Paper 1 . The model parameters $P_{1}$ and $P_{3}$ ranged from 0.05 to 1.25 at intervals of 0.05 , and $P_{2}$ was taken as 0.1 for the case of a strongly unstable frictional property, 0.5 for an unstable frictional property, and 1.0 for a frictional property at the boundary between stable and unstable.

The initial conditions were $V_{\text {init }}=0.001 V_{\mathrm{pl}}$ and $\theta_{\text {init }}=$ $L / V_{\text {init }}$ for all three blocks. In order to avoid transient characteristics in the results because of the initial conditions used, we employed the results obtained after running the model for 40000 years.

\section{Results}

\subsection{Parameter dependency of slip behaviour}

In order to understand the dependence of slip behaviour on the model parameters, we plotted the periodicity of the slip events and the relative frequency of the aseismic slip events on the parameter plane $P_{1}-P_{3}$. Periodicity was examined as follows: slip events at each block were identified when the slip velocity exceeded $V_{\mathrm{pl}}$ and the time intervals between successive slip events were known. Histograms of the times between events were constructed using a bin size of $\mathrm{d} t$. For instance, when slip events repeatedly occurred at a constant interval of $t_{0}$, only the frequency at the bin including $t_{0}$ was nonzero. When a period- $n$ cycle of slip events occurred (where a period- $n$ cycle denotes that $n$ slip events with different recurrence intervals were included in a single period), the frequencies at $n$ bins were the same and zero at the other 
bins. We thus measured the number $N_{\mathrm{b}}$ of bins of nonzero frequencies, and $N_{\mathrm{b}}$ was then used to represent the periodicity. The bin size $\mathrm{d} t$ was 0.01 year, which is smaller than $0.1 \%$ of the average recurrence interval of slip events (50200 years). When $N_{\mathrm{b}}>64$, we regard the slip pattern as having no periodicity (hereafter: aperiodic).

Figure 2 shows the periodicity of slip events in Block 2 on the parameter plane $P_{1}-P_{3}$, where the periodicity is shown by a binary logarithmic colour scale ranging from 1 to 64 . The periodicities of Blocks 1 and 3 are almost the same as that of Block 2. The results indicate that: (1) aperiodic slip patterns appear for broader ranges of parameter space for the case of $K=1.0$ than for $K=0.2$, and (2) aperiodic slip patterns tend to occur for $P \sim 1.00$, which is in the neighbourhood of the stable-unstable boundary of frictional behaviour. Both observations are consistent with the results of the twoblock system (Paper 1).

We then calculated the ratio $R_{\mathrm{a}}$ of the number of aperiodic cases to the total number of cases for each phase diagram. The $R_{\mathrm{a}}$ values obtained are shown in Fig. 2. $R_{\mathrm{a}}$ tends to increase with $K$ or $P_{2}$. In order to compare the present threeblock system with the two-block system, we conducted simulations of the two-block system using the same model as Paper 1 for the parameters $0.05 \leq\left(k_{0}+k_{12}\right) / k_{\mathrm{c} 1} \leq 1.25,0.05 \leq$ $\left(k_{0}+k_{12}\right) / k_{\mathrm{c} 2} \leq 1.25$, and $k_{12} / k_{0}=0.2$ and 1.0 , where $k_{\mathrm{ci}}$ is the critical stiffness of the $i$ th block in the two-block system. The values of $R_{\mathrm{a}}$ are $19.1 \%$ for $k_{12} / k_{0}=0.2$ and $66.8 \%$ for $k_{12} / k_{0}=1.0$ for the two-block system, while the $R_{\mathrm{a}}$ values are $15.4-70.4 \%$ for $k_{12} / k_{0}=0.2$ and $69.5-85.2 \%$ for $k_{12} / k_{0}=1.0$ for the three-block system. $R_{\mathrm{a}}$ in the threeblock system is generally larger than in the two-block system, indicating an increase in complexity with the increase in the number of degrees of freedom from 2 to 3 .

In most cases, the maximum slip velocity at the $i$ th block decreases as $P_{i}$ increases. Here we define "seismic slip" to be slip with $\log \left(V / V_{\mathrm{pl}}\right)>8$, which corresponds to $V \gtrsim$ $0.13 \mathrm{~m} \mathrm{~s}^{-1}$. A slip event with the maximum slip velocity lower than this value is regarded as an "aseismic slip" event. Changes in the ratio of the number of aseismic slip events to the total number of slip events for $K=0.2$ and $K=1.0$ are shown in Figs. 3 and 4, respectively. When the interaction is strong ( $K=1.0$ ), aseismic slip events occur for wider ranges of $P_{1}$ and $P_{3}$ for $P_{2} \leq 0.5$. A similar tendency is observed in the two-block system, as discussed in Paper 1. In addition, the ratio of aseismic slip events is always less than $100 \%$ for $K=1.0$, indicating that seismic slip events occur for the overall range tested. Even when $P_{1}$ and $P_{3}$ are larger than 1.0, seismic slip events occur at Blocks 1 and 3, triggered by a seismic slip event at Block 2 because of strong coupling. The parameter study for the two-block system (Paper 1) showed that the occurrence of aseismic slip events complicated the slip behaviour of the blocks. This finding is consistent with the result from the three-block system that an aperiodic slip pattern is common for $P_{i}>1.0$ (Fig. 2).
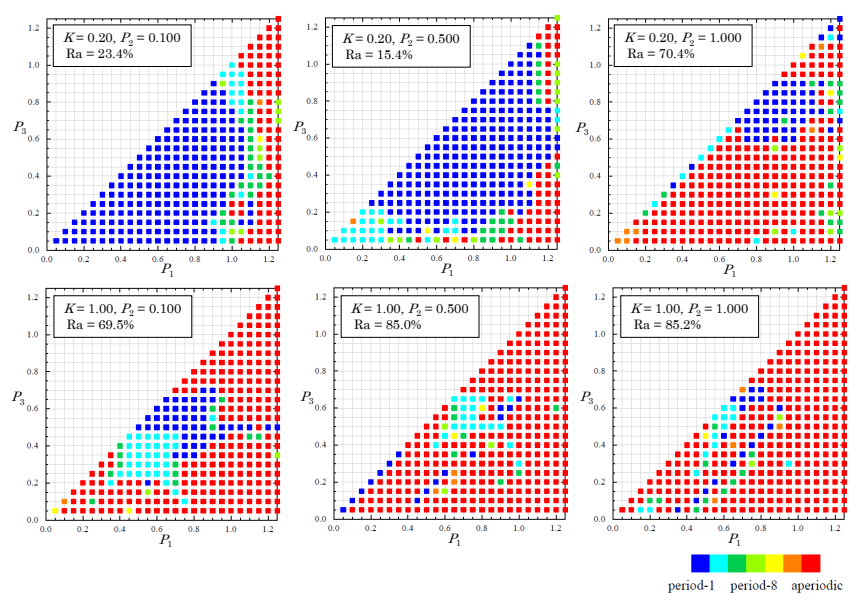

Figure 2. Parameter dependency of periodicity of slip events in Block 2 on $P_{1}$ and $P_{3}$ coordinates for $K=0.20$ (a-c) and $K=1.00$ (d-f) and $P_{1}=0.100,0.500$, and 1.000 . The periodicities for parameter sets are discretely plotted using seven colours, which indicate period-1, 2-3, 4-7, 8-15, 16-31, 32-63, and aperiodic ( $\geq 64)$. See text for the definition of $R_{\mathrm{a}}$.
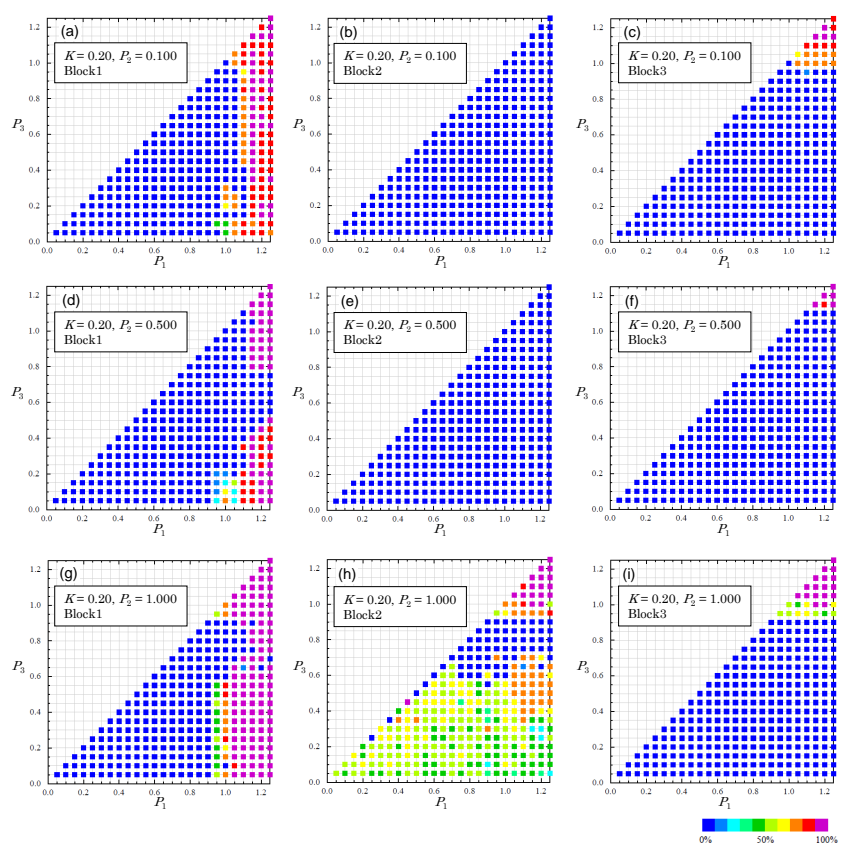

Figure 3. The ratio of aseismic slip events to total slip events for the case of $K=0.20$.

In order to examine the effect of the initial conditions on the simulation results, we conducted a parameter study with random initial conditions, where the different $V_{\text {init }}$ for the three blocks were varied within a range of $10 \%$ for each run. We confirmed that the periodicities of slip events (Fig. 2) for various initial conditions were unchanged for simulated slip histories after running the simulation for 40000 years. This is because slip behaviour for periodic slip patterns finally 

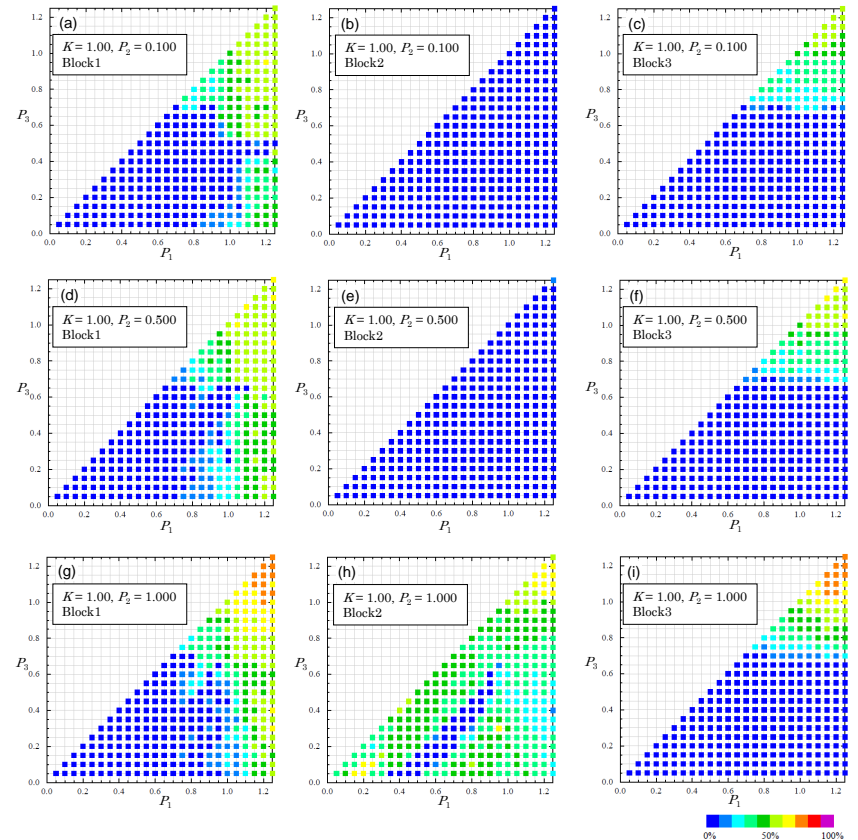

Figure 4. The ratio of aseismic slip events to total slip events for the case of $K=1.00$.

becomes the same after a transitional period independent of the initial conditions. The ratio of the aseismic slip events to the total slip events was changed by about $5 \%$ at most for each aperiodic case, and the patterns in Figs. 3-4 were only slightly dependent on the initial conditions.

\subsection{Complex slip pattern}

The complex slip patterns observed in the present three-block system are classified into five types according to the slip mode, the variability of the recurrence interval of slip events, and the intermittency. Figure 5 shows the parameter regions where the five complex slip patterns occur in phase diagrams, showing that the occurrence of a slip pattern depends on the parameters used. For each parameter set, we calculated the average value $T_{\text {ave }}$ and the standard deviation $\delta T$ of the recurrence intervals of the seismic slip events in Block 2, and took the coefficient of variation $\mathrm{COV}=\delta T / T_{\text {ave }}$. Then we obtained the averages of $T_{\text {ave }}$ and COV from all the samples for each complex slip pattern. The values $\overline{T_{\text {ave }}}$ and $\overline{\mathrm{COV}}$ are shown in Table 1 together with the order of the slip events in the three blocks and the intermittency of the slip patterns. To obtain $\overline{T_{\text {ave }}}$ and $\overline{\mathrm{COV}}$, we used data for seismic slip events in Block 2. This is because many aseismic slip events occur in Blocks 1 and 3 in some cases, and the time interval between seismic slip events is sometimes too long to be used for statistical analysis. We examine the characteristics of the five complex slip patterns described below by showing example time histories of slip velocity $V$ and frictional coefficient $\mu-\mu_{*}$.
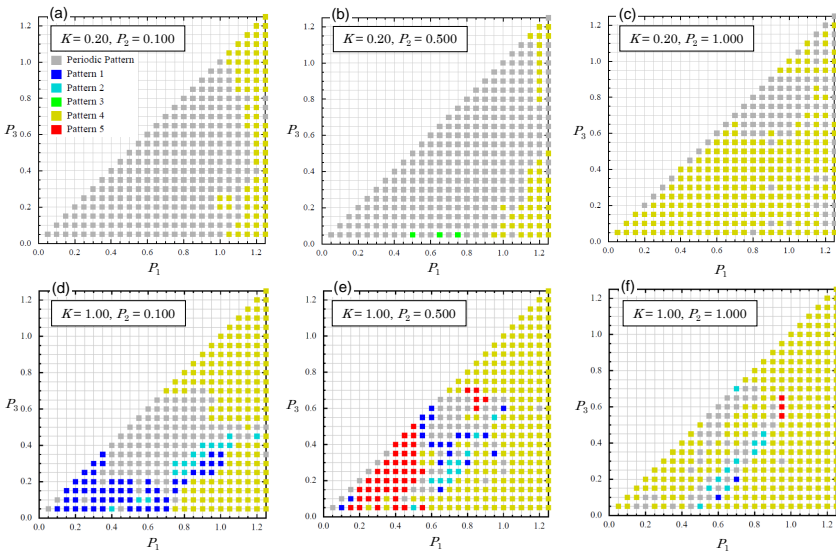

Figure 5. Phase diagrams of slip patterns for the three-block system. Periodic patterns are plotted with grey points and the others, which correspond to red points in Fig. 2, are classified into five complex patterns as described in Table 1 .

\subsubsection{Pattern 1}

Pattern 1 is a complex slip pattern that contains only seismic slip events. The slip events seem to occur randomly and the variation in the recurrence interval is relatively large. The order of the slip events in the three blocks is not constant. Figure $6 \mathrm{a}$ and $\mathrm{b}$ show example histories of $V$ and $\mu-\mu_{*}$ for pattern 1 . The bifurcation diagram of recurrence intervals of slip events for $K=1.00, P_{1}=0.450, P_{2}=0.100$, and $0.15 \leq P_{3} \leq 0.35$ is shown in Fig. 7 , in which the recurrence intervals of slip events at Block 1 are plotted against $P_{3}$. Figure 7 shows that a period-2 slip pattern occurs for $P_{3} \geq$ 0.243 , a period-6 pattern occurs for $0.238 \leq P_{3}<0.243$ and a period-12 pattern occurs for $0.229 \leq P_{3}<0.238$, indicating an increase in complexity with decreasing $P_{3}$. Finally, the slip pattern becomes aperiodic.

\subsubsection{Pattern 2}

Pattern 2 is an aperiodic pattern with only seismic slip events. Example histories of $V$ and $\mu-\mu_{*}$ for pattern 2 are shown in Fig. 8a and b, respectively. The order of slip events in the three blocks is the same in each case. For example, in the case shown in Fig. 8, Block 3 always slips first, Block 2 second, and Block 1 third in each sequence. The delay times between the slip events at Blocks 3 and 2 are shorter than 1 year and those between Blocks 2 and 1 vary from 3 to 15 years. Although this behaviour is apparently periodic, it is regarded as aperiodic from the analysis of recurrence intervals (Fig. 2). The COVs of recurrence intervals of slip events for pattern 2 are about one sixth of those for pattern 1 (Table 1). This behaviour is called "quasiperiodic", in which the trajectory winds around endlessly on the torus, never intersecting with itself and yet never quite closing (Strogatz, 1994). The bifurcation diagram of recurrence intervals for pattern 2 (Fig. 9) 

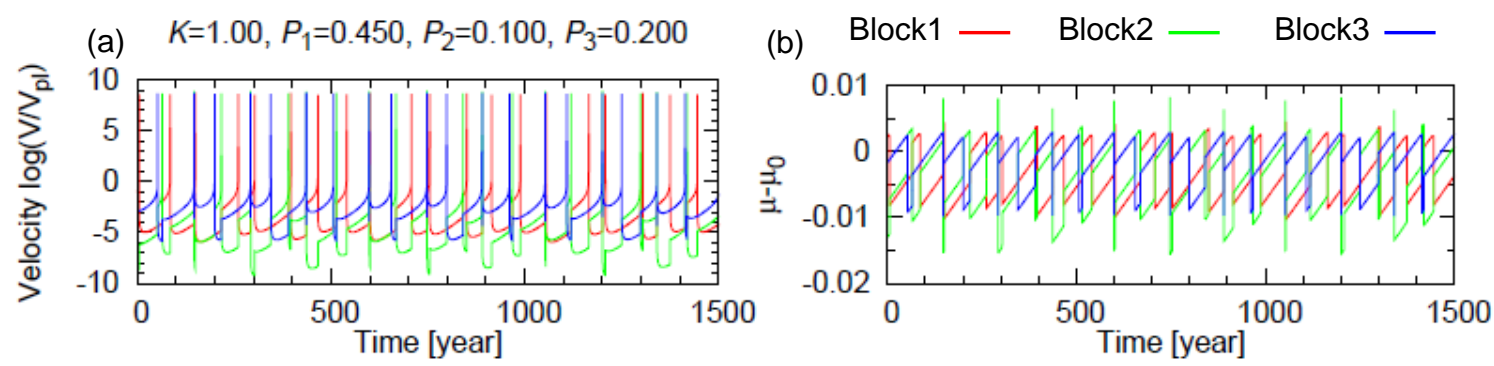

Figure 6. Example histories of (a) $V$ and (b) $\mu-\mu_{*}$ for pattern 1 . The model parameters are set to $K=1.00, P_{1}=0.450, P_{2}=0.100$, and $P_{3}=0.200$.

Table 1. The slip mode, the average value of the recurrence interval $\overline{T_{\text {ave }}}$ and the coefficient of variation $\overline{\mathrm{COV}}$, the order of slip events in the three blocks, and the intermittency of the slip pattern for the five slip patterns. Data for seismic slip events in Block 2 for all cases categorized into each complex slip pattern are used for $\overline{T_{\mathrm{ave}}}$ and $\overline{\mathrm{COV}}$. See text for details of calculation.

\begin{tabular}{llccll}
\hline Pattern & Slip mode & $\overline{T_{\text {ave }}}$ & $\overline{\mathrm{COV}}$ & $\begin{array}{l}\text { Order of } \\
\text { slip events }\end{array}$ & Intermittency \\
\hline 1 & Seismic & $72.9 \pm 8.6$ & $0.210 \pm 0.098$ & Variable & No \\
2 & Seismic & $71.7 \pm 6.7$ & $0.034 \pm 0.018$ & Invariable & No \\
3 & Seismic & $117.5 \pm 5.4$ & $0.246 \pm 0.016$ & Variable & Yes \\
4 & Seismic/aseismic & $109.1 \pm 49.0$ & $0.462 \pm 0.238$ & Variable & No \\
5 & Seismic/aseismic & $58.7 \pm 8.0$ & $0.390 \pm 0.086$ & Variable & Yes \\
\hline
\end{tabular}

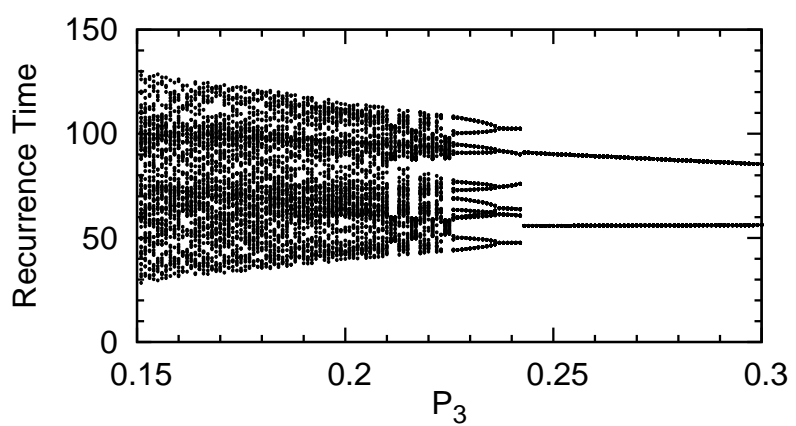

Figure 7. Bifurcation diagram of recurrence intervals of slip events at Block 1 near the transition boundary between multiperiodic patterns and complex pattern 1 . The model parameters are $K=1.00$, $P_{1}=0.450$, and $P_{2}=0.100$, and $P_{3}$ ranges from 0.15 to 0.35 .

is typically found to show a bifurcating to quasiperiodic pattern (Albers and Sprott, 2006). As $P_{3}$ decreases, the recurrence pattern changes from period- 1 to pattern 2 through a period-adding sequence, in which the number of periods increases monotonically. As $P_{3}$ further decreases, the recurrence pattern changes to become more complex with seismic and aseismic slip events, which is defined as pattern 4 . The sensitivity of the trajectory to small perturbations of initial conditions for pattern 2 is discussed in Sect. 4.2.

\subsubsection{Pattern 3}

Example histories of $V$ and $\mu-\mu_{*}$ for pattern 3 are shown in Fig. 10; here only seismic slip events occur and the order of slip events in the three blocks is variable. The average recurrence intervals of the slip events at the three blocks differ, and a slip event at a block may be skipped in a sequence. For instance, four seismic slip events occur in Block 1 and three seismic slip events occur in Blocks 2 and 3 during the time interval between 100 and 600 years in Fig. 10. Pattern 3 occurs for $K=0.20$ (Fig. 5b), in contrast to the result for the twoblock system, in which an aperiodic pattern of seismic slip events is not observed for $K=0.20$ (Paper 1). Two different recurrence patterns occur alternately with irregular durations, showing intermittent behaviour as discussed in Sect. 4.1. The transition from a periodic pattern to pattern 3 happens suddenly, as shown in a bifurcation diagram of the recurrence interval for pattern 3 (Fig. 11). A multiperiodic recurrence pattern suddenly changes to a complex recurrence pattern at $P_{3}=0.0395$. Similar sudden bifurcations occur at the transition points of patterns 4 and 5 .

\subsubsection{Pattern 4}

Pattern 4 is an aperiodic slip pattern with seismic and aseismic slip events as shown in Fig. 12, which shows example histories of $V$ and $\mu-\mu_{*}$. The order of the slip events in the blocks is highly variable. In most cases of pattern 4 , the COV of the recurrence intervals of seismic slip events is larger than 
(a) $K=1.00, P_{1}=0.650, P_{2}=0.500, P_{3}=0.200$

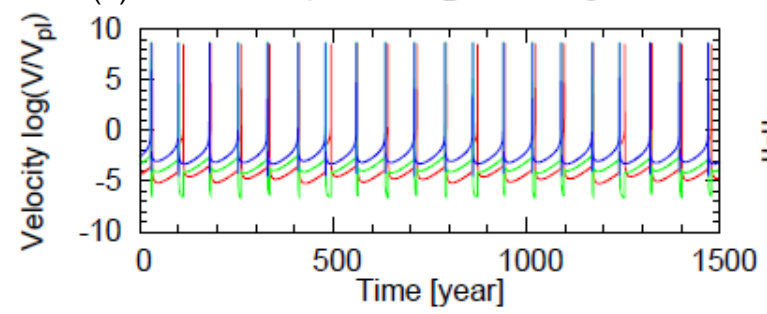

(b) Block1 - Block2 - Block3 -

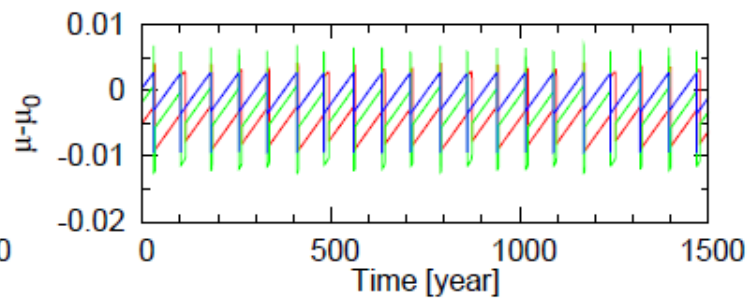

Figure 8. Example histories of (a) $V$ and (b) $\mu-\mu_{*}$ for pattern 2 . The model parameters are set to $K=1.00, P_{1}=0.650, P_{2}=0.500$, and $P_{3}=0.200$.

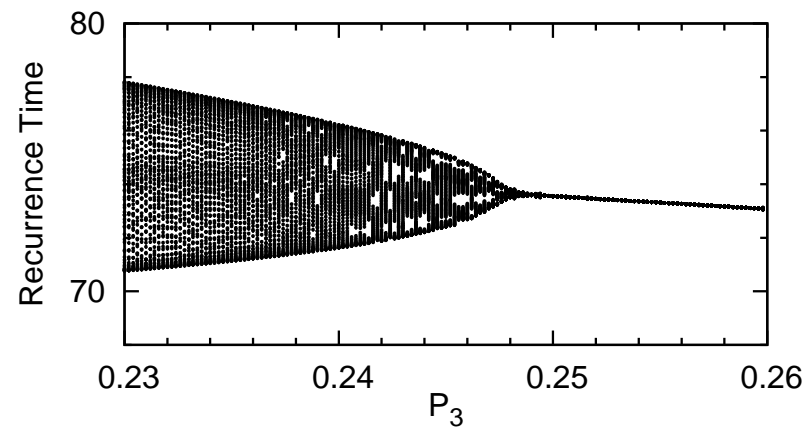

Figure 9. Bifurcation diagram of recurrence intervals of slip events at Block 1 near the transition to pattern 2 . The model parameters are $K \quad=1.00, P_{1}=0.650$, and $P_{2}=0.500$, and $0.23 \leq \quad P_{3}<0.26$.

those of patterns 1-3 with only seismic slip events (Table 1). Pattern 4 is the most common complex slip pattern and it tends to occur when the frictional property of at least one of the blocks is close to the stable-unstable transition boundary, i.e. $P_{i} \sim 1.0$ (Fig. 5).

\subsubsection{Pattern 5}

Pattern 5 is also an aperiodic slip pattern that includes both seismic and aseismic slip events. Figure 13 shows example histories of $V$ and $\mu-\mu_{*}$. Pattern 5 is characterized by two different slip behaviours that appear alternately: one is aperiodic behaviour with little variation in the recurrence interval and the other is aperiodic behaviour with greater variation in the recurrence interval. In the period from 0 to 1000 years, for which simulated histories are enlarged in Fig. 13c and d, only seismic slip events occur. The recurrence interval of seismic slip events at Block 2 during this period is $39.5 \pm 3.3$ years. The order of slip events in the three blocks is constant in this period. In the period from 1500 to 2200 years, for which simulated histories are enlarged in Fig. 13e and f, both seismic and aseismic slip events occur. The recurrence interval of seismic slip events at Block 2 during this period is $42.4 \pm 18.3$ years. This large variation in recurrence interval is caused by the frequent and random occurrence of aseismic slip events, which partially release stress and significantly prolong the time intervals between seismic slip events. The order of seismic slip events in the three blocks changes randomly.

\section{Discussion}

\subsection{Temporal evolution of recurrence interval}

We examine the variation of the recurrence interval $T_{n}$ with time, where $T_{n}$ is the time interval between the $(n-1)$ th and the $n$th seismic slip events. Figure 14 shows examples of the variation of $T_{n}$ at the three blocks for patterns 3 and 5 . The model parameters for the examples in Fig. 14a-f are the same as those for Figs. 10 and 13.

In the case shown in Fig. 14a (pattern 3), two different recurrence patterns alternate intermittently at Block 1 . In the first pattern, the recurrence interval monotonically increases from about 110 to about 160 years, and then decreases to about 110 years. In the other pattern, the recurrence interval oscillates between about 110 and 130 years. We call the former pattern $3 \mathrm{~A}$ and the latter $3 \mathrm{~B}$, and the time intervals of the two patterns are indicated by bars in Figs. 14a-c. The slip patterns at Blocks 2 and 3 also change intermittently and the time intervals of the two recurrence patterns almost coincide with those of Block 1 . The durations of pattern $3 \mathrm{~A}$ are nearly constant, while those of pattern $3 \mathrm{~B}$ are not constant. Figure 15a shows the iteration maps of $T_{n}$ of seismic slip events at the three blocks for the case shown in Figs. 14ac. Two orbits, which correspond to patterns $3 \mathrm{~A}$ and $3 \mathrm{~B}$, are observed for Block 1 (Fig. 14a).

Two different recurrence patterns are also observed for pattern 5, as shown in Fig. 14d-f (see also Fig. 13). The first is an aperiodic pattern with little variation in the recurrence interval (pattern 5A), where $T_{n}$ varies within a narrow range, and the second is an aperiodic pattern with greater variation in the recurrence interval (pattern 5B). Aseismic slip events are included only in the time intervals of pattern $5 \mathrm{~B}$ (Fig. 13e and f). To distinguish patterns 5A and 5B quantitatively, we measure $\left|T_{n}-T_{n-1}\right|$ for Block 1 . We regard $\left|T_{n}-T_{n-1}\right| \leq 10$ years as pattern $5 \mathrm{~A}$. In contrast, pattern 5B is assigned for $\left|T_{n}-T_{n-1}\right|>10$ years. The durations of patterns $5 \mathrm{~A}$ and $5 \mathrm{~B}$ are not constant. In each time interval of 
(a) $K=0.20, P_{1}=0.500, P_{2}=0.500, P_{3}=0.050$

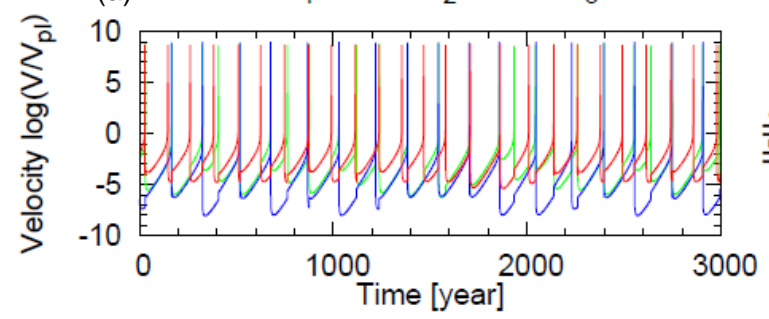

(b) Block1 - Block2 - Block3 -

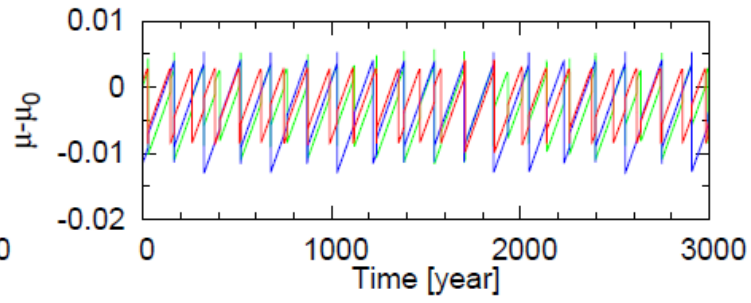

Figure 10. Example histories of (a) $V$ and (b) $\mu-\mu_{*}$ for pattern 3 . The model parameters are set to $K=0.20, P_{1}=0.500, P_{2}=0.500$, and $P_{3}=0.050$.

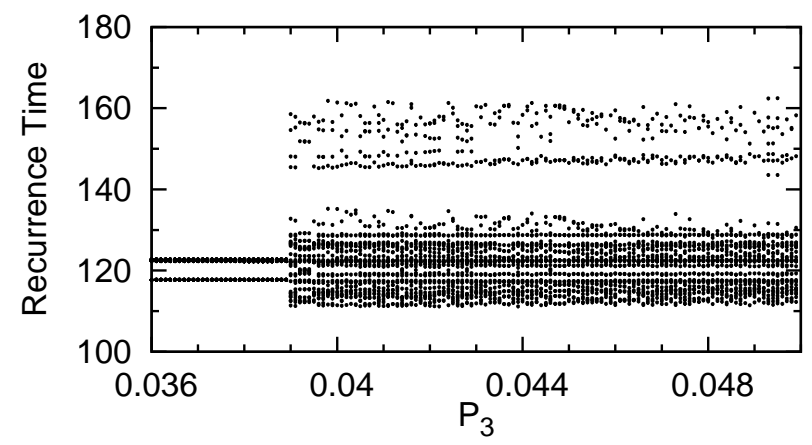

Figure 11. Bifurcation diagram of recurrence intervals of slip events at Block 1 near the transition boundary between periodic patterns and complex pattern 3 . The model parameters are $K=0.20, P_{1}=$ 0.500 , and $P_{2}=0.500$, and $P_{3}$ ranges from 0.036 to 0.05 .

pattern $5 \mathrm{~A}$, the fluctuation of $T_{n}$ is small at first before gradually increasing. Finally, $T_{n}$ suddenly jumps and the pattern changes to $5 \mathrm{~B}$. The time intervals for patterns $\mathrm{A}$ and $\mathrm{B}$ at Blocks 2 and 3 coincide with those of Block 1. The iteration maps of the recurrence intervals of seismic slip events for the periods from 200 to 1500 years and from 2500 to 3200 years in Fig. 14d-f are shown in Fig. 15b and c, respectively. Figure $15 \mathrm{~b}$ mostly corresponds to pattern $5 \mathrm{~A}$ and Fig. $15 \mathrm{c}$ to pattern 5B. In Fig. 15b and c, the start and end points of patterns $5 \mathrm{~A}$ and $5 \mathrm{~B}$ are indicated by arrows. Figure $15 \mathrm{~b}$ clearly shows that the variation in $T_{n}$ increases as the system approaches the transition point from pattern $5 \mathrm{~A}$ to $5 \mathrm{~B}$ for the three blocks. The maps in Fig. 15c are irregular, in contrast with those in Fig. 15b. Precursory behaviour for the transition from pattern $5 \mathrm{~B}$ to $5 \mathrm{~A}$ cannot be found.

Figure 16 shows the cumulative frequencies of the durations $\tau$ of patterns 3B, 5A, and 5B for the cases shown in Figs. 10 and 13. The frequencies of $\tau$ are approximately expressed by exponential functions, rather than the power functions observed for "on-off intermittent earthquake occurrence" (Bottiglieri and Godano, 2007). On-off intermittent earthquake occurrence is characterized by periods of clustered occurrence of earthquakes and relatively inactive periods for the times between successive clusters, which are regarded as "burst" and "laminar" phases, respectively. Bot- tiglieri and Godano (2007) obtained a power law distribution for the duration of laminar phases from the Southern California Catalogue. For pattern 3B, the frequencies of $\tau$ of multiples of 120 years, and of $\tau \sim 1440$ years in particular, are high (Fig. 16a). This is because the recurrence intervals of slip events during pattern 3B fluctuate around 120 years. In contrast, the distribution functions of duration for patterns $5 \mathrm{~A}$ and $5 \mathrm{~B}$ are more continuous than that of $3 \mathrm{~B}$.

\subsection{Lyapunov exponent}

In order to quantify the complexity of aperiodic slip patterns in the present three-block system, we calculated the Lyapunov exponent, which is a quantity representing the sensitivity of a system to small perturbations (Drazin, 1992). In particular we are interested in patterns 3 and 5 because apparently they are "intermittent chaos", in which irregular alternation of phases of different complex behaviour occurs (Paladin and Vulpiani, 1987). Nakanishi (1991) conducted a numerical simulation of an earthquake cycle using a cellular automaton version of a multi-spring-block model (Burridge and Knopoff, 1967). In his model, the Lyapunov exponent is positive when the frequency-magnitude relationship obeys a power law, and it is close to zero when the frequencymagnitude distribution has a characteristic peak, which may correspond to the characteristic earthquake model. Crisanti et al. (1992) used the same cellular automaton model as Nakanishi (1991) and examined the evolution of the Lyapunov exponent to characterize the degree of intermittency.

We calculated the Lyapunov exponent using an algorithm essentially the same as those of Nakanishi (1991) and Crisanti et al. (1992). Firstly, two trajectories $f_{\mathrm{A}}$ and $f_{\mathrm{B}}$ are calculated from slightly different initial conditions. We define the distance of the trajectories from the difference in the frictional coefficients as

$\delta\left(t_{n}\right)=\left(\sum_{i=1}^{3}\left(\mu_{i}^{\mathrm{A}}\left(t_{n}^{\mathrm{A}}\right)-\mu_{i}^{\mathrm{B}}\left(t_{n}^{\mathrm{B}}\right)\right)^{2}\right)^{1 / 2}$,

where $\mu_{i}^{\mathrm{A}}$ and $\mu_{i}^{\mathrm{B}}$ are the frictional coefficients of the $i$ th block for $f_{\mathrm{A}}$ and $f_{\mathrm{B}}$, respectively, and $t_{n}^{\mathrm{A}}$ and $t_{n}^{\mathrm{B}}$ are the times just after the $n$th seismic slip events for trajectories $f_{\mathrm{A}}$ and 
(a) $K=1.00, P_{1}=0.650, P_{2}=0.500, P_{3}=0.050$

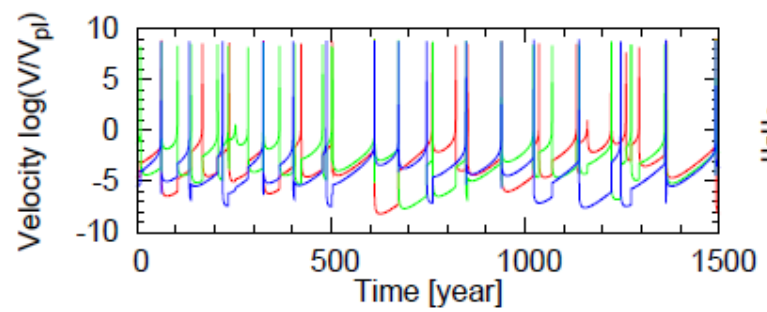

(b) Block1 - Block2 - Block3 -

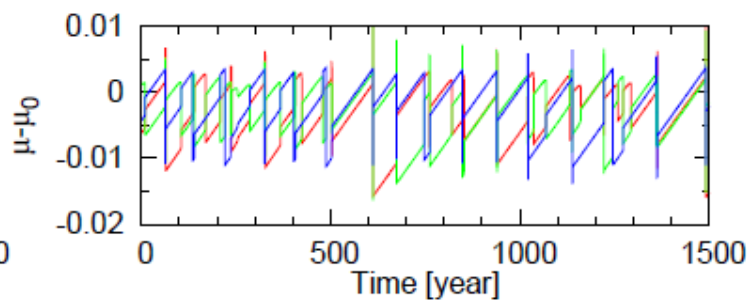

Figure 12. Example histories of (a) $V$ and (b) $\mu-\mu_{*}$ for pattern 4 . The model parameters are set to $K=1.00, P_{1}=0.650, P_{2}=0.500$, and $P_{3}=0.050$.

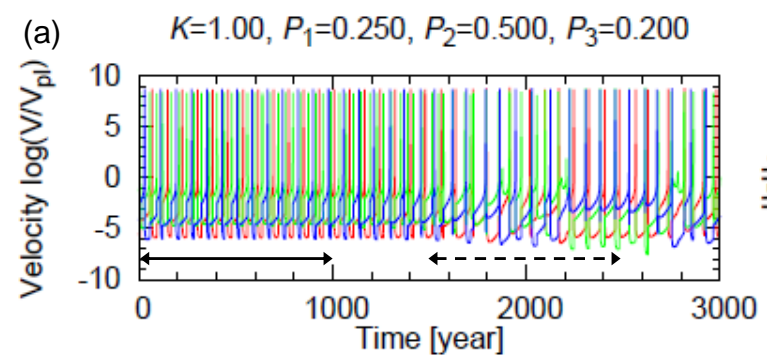

(c)

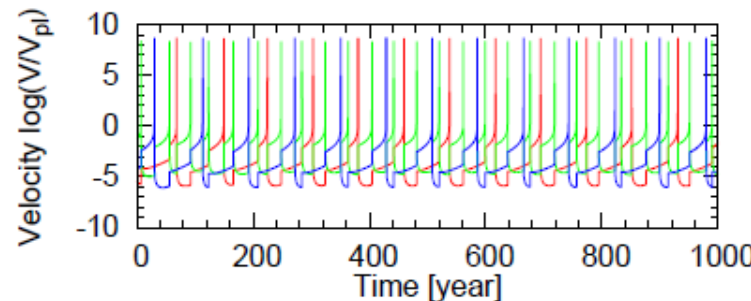

(e)

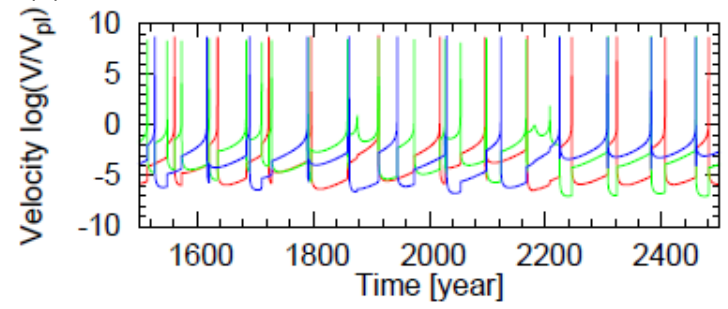

(b) Block1 $\quad$ Block2 - Block3 -

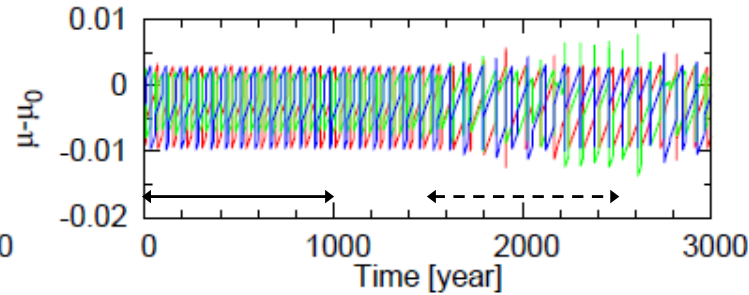

(d)

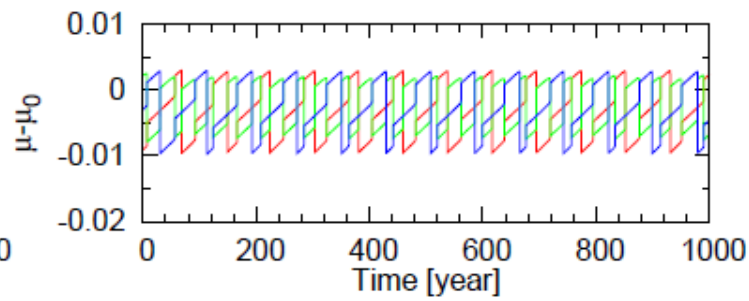

(f)

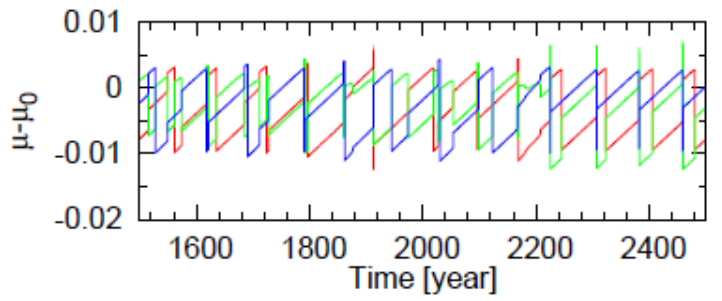

Figure 13. Example histories of (a) $V$ and (b) $\mu-\mu_{*}$ for pattern 5. The model parameters are set to $K=1.00, P_{1}=0.250, P_{2}=0.500$, and $P_{3}=0.200$. The sections indicated by the solid arrows in (a) and (b) are expanded in (c) and (d), and those indicated by the dashed arrows are expanded in (e) and (f).

$f_{\mathrm{B}}$, respectively. Note that in general $t_{n}^{\mathrm{A}} \neq t_{n}^{\mathrm{B}}$ because the occurrence time of seismic slip events depends on the initial conditions. The Lyapunov exponent $\lambda$ is defined by

$\lambda=\frac{1}{N} \sum_{n=1}^{N} \ln \frac{\delta\left(t_{n}\right)}{\delta_{0}}$,

where $\delta_{0}$ is the initial value of $\delta$. In the present study, we used $\delta_{0}=10^{-5}$.

In order to characterize the intermittency, we examined the variation of $\lambda$ with time. Because we are interested in the relationship between $\lambda(t)$ and the two patterns A and B, each $\lambda$ value is calculated by setting $N=5$, which is sufficiently smaller than the average numbers of slip events during the two patterns. Table 2 shows the average values and the standard deviations of $\lambda(t)$ for the example cases shown in Figs. 6, 8, 10, 12, and 13, which correspond to patterns $1-5$, respectively. Each value is calculated for periods of 100000 years. The negative values of $\lambda$ for patterns 1 and 2 indicate that the evolution of the system is stable for small perturbations and the complex slip behaviours in patterns 1 and 2 are not chaotic. The positive $\lambda$ values for patterns 3 , 4 , and 5 indicate chaotic behaviour, in which the evolution of slip is sensitive to small perturbations, and the long term 
Table 2. The average values and the standard deviations of the Lyapunov exponent $\lambda$ for the five slip patterns. The model parameters for patterns 1-5 are the same as those for Figs. 6, 8, 10, 12, and 13, respectively. Patterns 3 and 5 are calculated for time intervals including both patterns $\mathrm{A}$ and $\mathrm{B}$.

\begin{tabular}{lr}
\hline Pattern & $\lambda$ \\
\hline 1 & $-0.573 \pm 0.266$ \\
2 & $-1.678 \pm 0.227$ \\
3 & $0.725 \pm 0.798$ \\
4 & $0.712 \pm 0.942$ \\
5 & $0.483 \pm 0.986$ \\
\hline
\end{tabular}
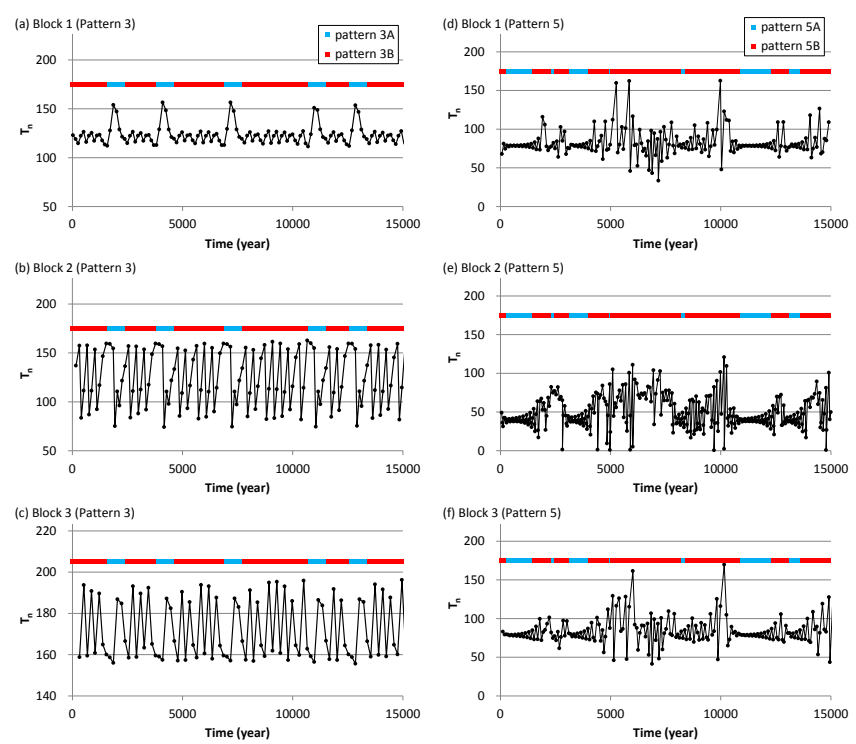

Figure 14. Examples of the variation of recurrence interval $T_{n}$ of slip events at the three blocks for (a)-(c) pattern 3 and (d)-(f) pattern 5. The time intervals of the two patterns $A$ and $B$ are indicated by blue and red bars, respectively. The model parameter sets for (a)-(c) and (d)-(f) are the same as those for Figs. 10 and 13, respectively.

forecasting of slip evolution is therefore practically impossible. Albers and Sprott (2006) calculated the Lyapunov exponents for a simulated time series of a time-delay neural network model around a bifurcation point from quasiperiodic to chaotic behaviour, indicating that the Lyapunov exponents take negative values for quasiperiodic states and are positive for chaotic states. Our result is consistent with that of Albers and Sprott (2006).

Figure 17 shows the evolution of $\lambda$ for the time intervals shown in Fig. 14 for the example cases of patterns 3 and 5. Each $\lambda$ value is plotted at the end of the time window for calculating $\lambda$ because it is useful for evaluating the predictability of a change in slip pattern due to change in $\lambda$. For both patterns 3 and 5, the boundaries between the intervals $A$ and $B$, which are defined in Sect. 4.1 and indicated by
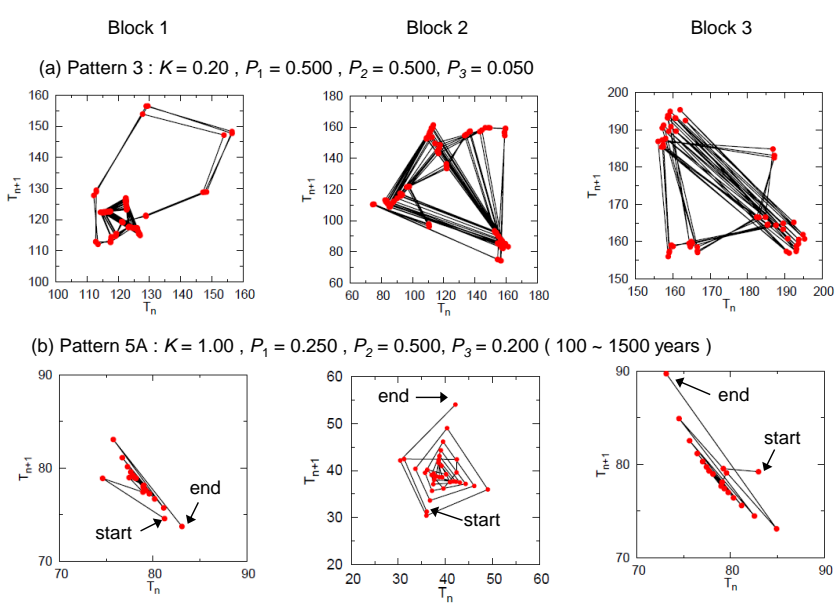

(c) Pattern 5B : $K=1.00, P_{1}=0.250, P_{2}=0.500, P_{3}=0.200(2500 \sim 3000$ years $)$
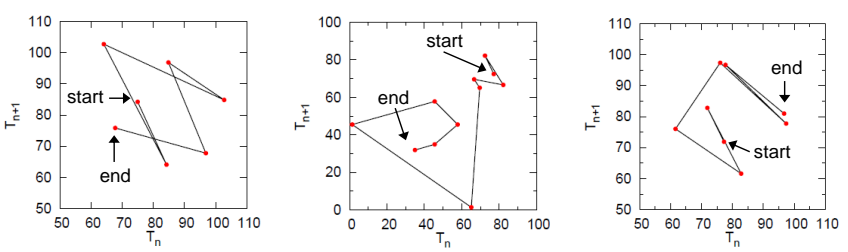

Figure 15. Iteration maps of recurrence intervals $T_{n+1}$ versus $T_{n}$ for (a) pattern 3, (b) pattern 5A, and (c) pattern 5B. The start and end points of maps (b) and (c) are indicated by arrows.
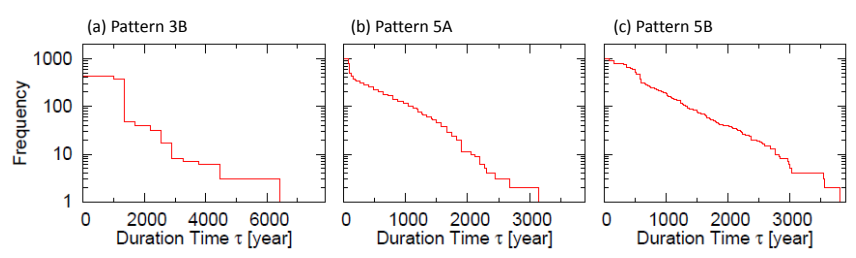

Figure 16. Frequencies of durations longer than $\tau$ for patterns (a) 3B, (b) 5A, and (c) 5B. The model parameters for patterns 3 and 5 are the same as those for Figs. 10 and 13, respectively.

bars in Fig. 17, are correlated with the change in $\lambda$. During a period of pattern $3 \mathrm{~A}, \lambda$ decreases from positive to negative, then increases from the local minimum value, and at the same time the slip pattern changes to pattern $3 \mathrm{~B}$. In pattern $3 \mathrm{~B}, \lambda$ increases to become positive, and is nearly constant for a certain duration. The slip pattern abruptly changes to $3 \mathrm{~A}$ without any precursor. Figure $17 \mathrm{~b}$ indicates that pattern $5 \mathrm{~A}$ is characterized by negative values of $\lambda$. In each period of pattern $5 \mathrm{~A}$, $\lambda$ gradually increases with time, which corresponds to the fluctuation of $T_{n}$ increasing with time (Fig. 14d). In contrast, the fluctuation of $\lambda$ during pattern $5 \mathrm{~B}$ is variable, as shown in Fig. $17 \mathrm{~b}$, and any change to pattern $5 \mathrm{~A}$ is therefore difficult to predict. Although we show only two example cases of the correlation between $\lambda$ and changes in slip pattern in patterns 3 and 5, similar correlations are observed for other cases with different parameter sets in patterns 3 and 5 . 

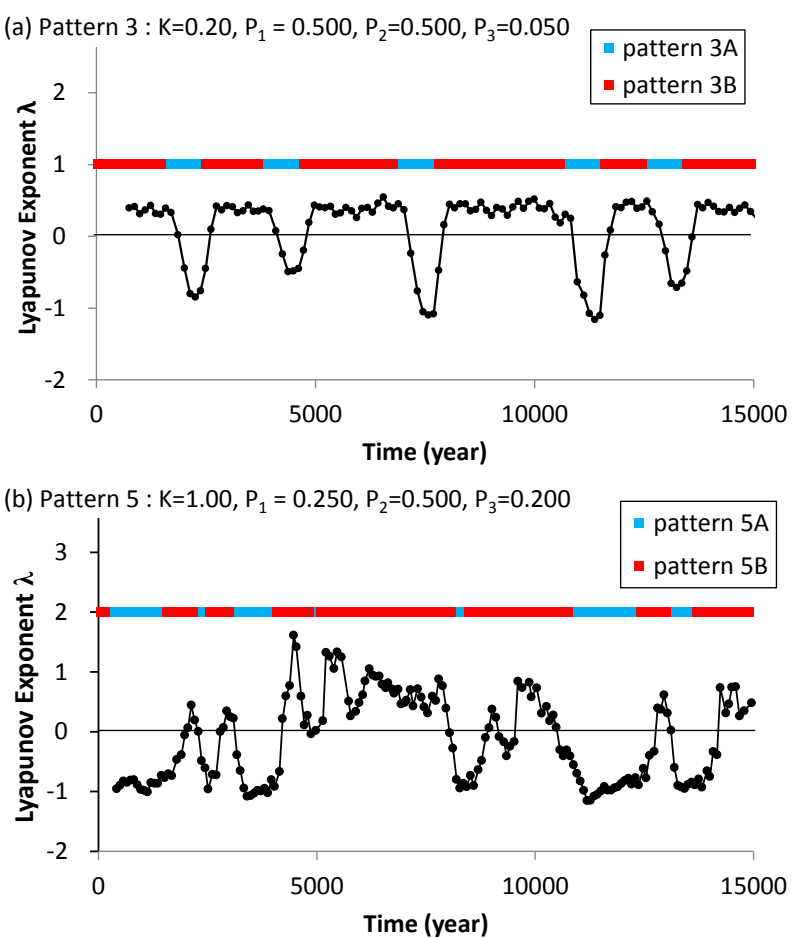

Figure 17. Examples of the variation of Lyapunov exponents $\lambda$ for patterns 3 and 5. $\lambda(t)$ is plotted at the ends of time windows where $\lambda(t)$ is calculated. The blue and red bars indicate the time periods of patterns A and B, respectively.

Intermittent chaos is not observed in the two-block system of Paper 1. Crisanti et al. (1992) found that the degree of intermittency, which is expressed by the variation of $\lambda$, increases with the number of interacting blocks. The fact that intermittent chaos is newly observed in the three-block system is consistent with the findings of Crisanti et al. (1992). The existence of intermittent chaos implies that patterns of earthquake occurrence may change abruptly from one to another. Similar abrupt changes in slip pattern were reported for a five-block system with rate- and state-dependent friction by Mitsui and Hirahara (2004), though they provided no quantitative analyses. Moreover, Kato et al. (2007) found changes in slip pattern in their multisegmented fault model in an elastic medium.

\subsection{Time- or slip-predictable model}

Whether the slip pattern obeys the time- or slip-predictable model is important for understanding the predictability of slip events (Shimazaki and Nakata, 1980). We examined the histories of the displacements of the three blocks from a single point of reference for patterns $1-5$. Figure 18a shows an example history of normalized displacement $X$ for pattern 1 corresponding to the case shown in Fig. $6 . X$ is defined by

$X=\left(x-x_{\min }\right) /\left(x_{\max }-x_{\min }\right)$,

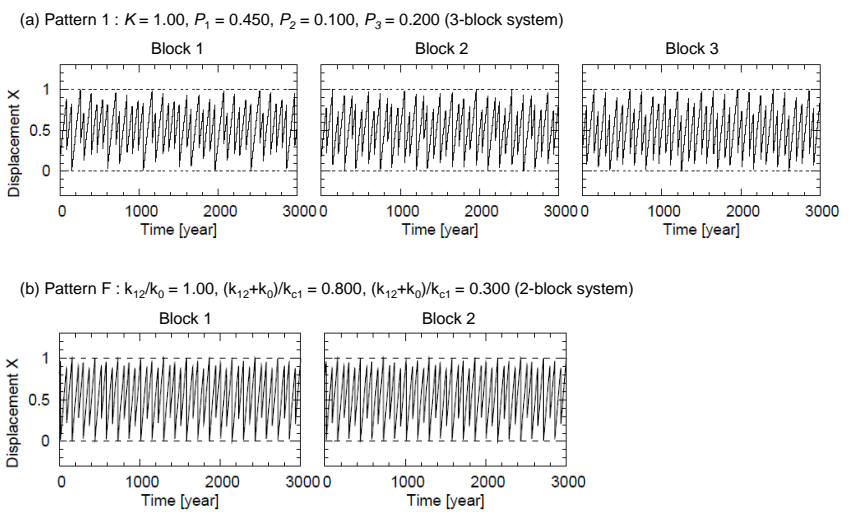

Figure 18. Example histories of the normalized displacement $X$ of the three blocks from the reference point for (a) pattern 1 in the three-block system and (b) pattern $\mathrm{F}$ in the two-block system (Paper 1). The model parameters for pattern 1 are the same as those relating to Fig. 6. The upper and lower broken lines indicate the time- and slip-predictable models, respectively.

where $x$ is the displacement from the reference point (Fig. 1), and $x_{\min }$ and $x_{\max }$ are the minimum and maximum values of $x$ in the observation period for each block. If slip events always occur when $X$ reaches the upper broken line $(X=1)$, the recurrence pattern can be explained by the timepredictable model. Meanwhile, if $X$ always reaches the lower broken line $(X=0)$ after slip events, the recurrence pattern can be explained by the slip-predictable model. To quantify the similarity to the two recurrence models, we examined the difference $X_{\mathrm{u}}$ between the upper broken line $(X=1)$ and the local maxima of $X$ before slip events, and $X_{1}$ between the lower broken line $(X=0)$ and the local minima of $X$ after slip events. To compare the simulated slip histories in the present three-block system with those in the two-block system, we used the simulation results reported in Paper 1 (Fig. 18b). Table 3 shows the average values $\overline{X_{\mathrm{u}}}$ and $\overline{X_{1}}$ for patterns $1-5$ and the periods of pattern $5 \mathrm{~A}$ for $0-15000$ year time periods, together with the $\overline{X_{\mathrm{u}}}$ and $\overline{X_{1}}$ values for patterns $D_{2}, E_{2}$, and $F$ in the two-block system (Paper 1) for comparison.

For pattern 2, both $\overline{X_{\mathrm{u}}}$ and $\overline{X_{1}}$ are small at the three blocks. The simulated history of $X$ for pattern 2 can be better explained by the time- and slip-predictable models than by the others, which is consistent with the small COV of the recurrence intervals (Table 1 ). For pattern $3, \overline{X_{\mathrm{u}}}$ is significantly smaller than $\overline{X_{1}}$ for Blocks 1 and 3, indicating that the recurrence patterns for Blocks 1 and 3 are approximately explained by the time-predictable model, and both $\overline{X_{\mathrm{u}}}$ and $\overline{X_{1}}$ are large at Block 2. For patterns 1, 4, and 5, both $\overline{X_{\mathrm{u}}}$ and $\overline{X_{1}}$ are large at the three blocks and neither the time- nor the slip-predictable model can explain the simulated slip histories at the three blocks. However, for the time period of pattern $5 \mathrm{~A}$, both $\overline{X_{\mathrm{u}}}$ and $\overline{X_{1}}$ are small at Blocks 1 and 3 and the 
Table 3. The average values of the displacement differences $\overline{X_{\mathrm{u}}}$ and $\overline{X_{1}}$ for slip patterns $1-5$ and an intermittent slip pattern (pattern 5A) in the three-block system, and patterns $\mathrm{D}_{2}, \mathrm{E}_{2}$, and $\mathrm{F}$ in the two-block system (Paper 1). See main text for the definitions of $\overline{X_{\mathrm{u}}}$ and $\overline{X_{1}}$. The model parameters for patterns $1-5$ are the same as those for Figs. 6, 8, 10, 12, and 13, respectively. The values are calculated for the time period 0-15000 years. Patterns 3 and 5 are calculated for time intervals including both patterns A and B. The model parameters for patterns $\mathrm{D}_{2}, \mathrm{E}_{2}$, and $\mathrm{F}$ are the same as those for Figs. $6 \mathrm{c}, 7 \mathrm{c}$, and 8 in Paper 1, respectively.

\begin{tabular}{|c|c|c|c|c|c|c|}
\hline \multirow[t]{2}{*}{ Pattern } & \multicolumn{2}{|c|}{ Block 1} & \multicolumn{2}{|c|}{ Block 2} & \multicolumn{2}{|c|}{ Block 3} \\
\hline & $\overline{X_{\mathrm{u}}}$ & $\overline{X_{1}}$ & $\overline{X_{\mathrm{u}}}$ & $\overline{X_{1}}$ & $\overline{X_{\mathrm{u}}}$ & $\overline{X_{1}}$ \\
\hline 1 & 0.152 & 0.196 & 0.145 & 0.158 & 0.148 & 0.122 \\
\hline 2 & 0.030 & 0.081 & 0.062 & 0.019 & 0.038 & 0.028 \\
\hline 3 & 0.035 & 0.192 & 0.116 & 0.160 & 0.016 & 0.110 \\
\hline 4 & 0.201 & 0.398 & 0.335 & 0.365 & 0.151 & 0.300 \\
\hline 5 & 0.221 & 0.327 & 0.343 & 0.369 & 0.168 & 0.371 \\
\hline $5 \mathrm{~A}$ & 0.021 & 0.019 & 0.093 & 0.117 & 0.033 & 0.036 \\
\hline $\mathrm{D}_{2}$ (two-block system) & 0.192 & 0.543 & 0.058 & 0.234 & - & - \\
\hline $\mathrm{E}_{2}$ (two-block system) & 0.171 & 0.458 & 0.021 & 0.083 & - & - \\
\hline F (two-block system) & 0.066 & 0.125 & 0.097 & 0.076 & - & - \\
\hline
\end{tabular}

recurrence pattern approximately accords with both the timeand slip-predictable models.

In the two-block system, the simulated slip histories for pattern F, where only seismic slip events occur, approximately agree with the time-predictable model, as discussed in Paper 1. Figure 18b shows example histories of the normalized displacement at the two blocks in pattern $\mathrm{F}$, for which corresponding histories of slip velocity and friction are shown in Fig. 8 in Paper 1. For patterns $\mathrm{D}_{2}$ and $\mathrm{E}_{2}$, where seismic and aseismic slip events occur, $\overline{X_{\mathrm{u}}}$ is small at Block 2, indicating that the recurrence patterns at Block 2 approximately accord with the time-predictable model. Although the simulated histories of displacements can in most cases be better approximated by the time-predictable model for the two-block system, many cases cannot be explained by either the time- or the slip-predictable model for the threeblock system. The increase in the number of connecting blocks from two to three complicates the recurrence patterns, leading to a reduction in predictability.

\section{Summary}

We used a three-degree-of-freedom spring-block model with a rate- and state-friction law to simulate earthquake cycles. We conducted a systematic parameter study to examine the periodicity of slip events and the occurrence of aseismic slip events. The range of parameters for which aperiodic slip patterns may be observed in the present three-block system is wider than that of the two-block system. An aperiodic slip pattern is observed for the condition of weak interaction $K=0.2$, in contrast to the absence of an aperiodic pattern for $K=0.2$ in the two-block system. The complex slip behaviour observed in the present three-block system can be classified into five slip patterns.
Intermittent chaos can be observed in the three-block system, where two different recurrence patterns of slip events occur alternately. The two different recurrence patterns of slip events are characterized by different values of the Lyapunov exponent. Although a change in the recurrence pattern can be predicted in some cases by considering the Lyapunov exponent, the prediction of a change in slip pattern is generally difficult. While intermittency has never previously been reported in studies of two-degree-of-freedom springblock models that assume various types of friction model (Huang and Turcotte, 1992; He, 2003; Yoshida and Kato, 2003), it was observed for a cellular automaton version of the three-degree-of-freedom spring-block model assuming simple static-dynamic friction (Nakanishi, 1991). This suggests that intermittency occurs depending on multiple interactions rather than on the complexity of the friction. An earthquake recurrence pattern may change in real earthquake fault systems quite suddenly when three or more faults interact. This implies difficulty for probabilistic earthquake forecasting based on several recurrences of earthquakes.

\section{The Supplement related to this article is available online at doi:10.5194/npg-21-841-2014-supplement.}

Acknowledgements. This study was supported by the Ministry of Education, Culture, Sports, Science and Technology (MEXT) of Japan, under its Observation and Research Program for Prediction of Earthquakes and Volcanic Eruptions.

Edited by: I. Zaliapin

Reviewed by: M. Naylor and one anonymous referee 


\section{References}

Abe, Y. and Kato, N.: Complex earthquake cycle simulations using a two-degree-of-freedom spring-block model with a rate- and state-friction law, Pure Appl. Geophys., 170, 745-765, 2012.

Albers, D. J. and Sprott, J. C.: Routes to chaos in high-dimensional dynamical systems: A qualitative numerical study, Physica D, 223, 194-207, 2006.

Ben-Zion, Y., Dahmen, K., Lyakhovsky, V., Ertas, D., and Agnon, A.: Self-driven mode switching of earthquake activity on a fault system, Earth Planet. Sc. Lett., 172, 11-21, 1999.

Bottiglieri, M. and Godano, C.: On-off intermittency in earthquake occurrence, Phys. Rev. E, 75, 026101, doi:10.1103/PhysRevE.75.026101, 2007.

Burridge, R. and Knopoff, L.: Model and theoretical seismicity, B. Seismol. Soc. Am., 57, 341-371, 1967.

Crisanti, A., Jensen, M. H., and Vulpiani, A.: Strongly intermittent chaos and scaling in an earthquake model, Phys. Rev. E, 46, 7363-7366, 1992.

Dahmen, K., Ertas, D., and Ben-Zion, Y.: Gutenberg-Richter and characteristic earthquake behavior in simple mean-field models of heterogeneous faults, Phys. Rev. E, 58, 1494-1501, 1998.

Dieterich, J. H.: Modeling of Rock Friction, 1, Experimental Results and Constitutive Equations, J. Geophys. Res., 84, 2161$2168,1979$.

Drazin, P. D.: Nonlinear systems, Cambridge University Press, 1992.

Erickson, B., Birnir, B., and Lavallee, D.: Periodicity, chaos and localization in a Burridge-Knopoff model of an earthquake with rate-and-state friction, Geophys. J. Int., 187, 178-198, 2011.

$\mathrm{He}, \mathrm{C}$. Interaction between two sliders in a system with rate- and state-dependent friction, Sci. China Ser. D, 46, 67-74, 2003.

Huang, J. and Turcotte, D. L.: Evidence for Chaotic Fault Interactions in the Seismicity of the San Andreas Fault and Nankai Trough, Nature, 348, 234-236, 1990.

Huang, J. and Turcotte, D. L.: Chaotic Seismic Faulting with a Mass-spring Model and Velocity-weakening Friction, Pure Appl. Geophys., 138, 569-589, 1992.

Kato, N., Lei, X., and Wen, X.: A synthetic seismicity model for the Xianshuihe fault, southernwestern China: simulation using a rate- and state-dependent friction law, Geophys. J. Int., 169, 286300,2007

Lyakhovsky, V., Ben-Zion, Y., and Agnon, A.: Earthquake cycle, fault zones, and seismicity patterns in a rheologically layered lithosphere, J. Geophys. Res., 106, 4103-4120, 1999.
Ma, S. and He, C.: Period Doubling as a Result of Slip Complexities in Sliding Surfaces with Strength Heterogeneity, Tectonophysics, 337, 135-145, 2001.

Mitsui, N. and Hirahara, K.: Simple Spring-mass Simulation of Earthquake Cycle along the Nankai Trough in Southwest Japan, Pure Appl. Geophys., 161, 2433-2450, 2004.

Nakanishi, H.: Statistical properties of the cellular-automaton model for earthquakes, Phys. Rev. A, 43, 6613-6621, 1991.

Nomanbhoy, N. and Ruff, L. J.: A simple discrete element model for large multiplet earthquakes, J. Geophys. Res., 101, 5707-5723, 1996.

Nussbaum, J. and Ruina, A.: A two degree-of-freedom earthquake model with static/dynamic friction, Pure Appl. Geophys., 125, 629-656, 1987.

Paladin, G. and Vulpiani, A.: Anomalous scaing laws in multifractal objects, Phys. Rep., 156, 147-225, 1987.

Pomeau, Y. and Manneville, P.: Intermittent transition to turbulence in dissipative dynamical systems, Commun. Math. Phys., 74, 189-197, 1980.

Press, W. H., Teukolsky, S. A., Vetterling, W. T., and Flannery, B. P.: Numerical Recipes in C: The Art of Scientific Computing, 2nd Edn., Cambridge Univ. Press, Cambridge, UK, 1992.

Ruina, A.: Slip instability and State Variable Friction Laws, J. Geophys. Res., 88, 10359-10370, 1983.

Sándor, B., Járai-Szabó, F., Tél, T., and Néda, Z.: Chaos on the conveyor belt, Phys. Rev. E, 87, 042920, doi:10.1103/PhysRevE.87.042920, 2013.

Schwartz, S. Y. and Rokosky, J. M.: Slow slip events and seismic tremor at circum-pacific subduction zones, Rev. Geophys., 45, RG3004, doi:10.1029/2006RG000208, 2007.

Shimazaki, K. and Nakata, T.: Time-predictable recurrence model for large earthquake, Geophys. Res. Lett., 7, 279-282, 1980.

Strogatz, S. H.: Nonlinear dynamics and chaos, Addison Wesley, 1994.

Weldon, R., Fumal, T., and Biasi, G.: Wrightwood and the earthquake cycle: what a long recurrence record tells us about how faults works, GSA Today, 14, 4-10, 2004.

Wesnousky, S. G.: The Gutenberg-Richter or characteristic earthquake distribution, which is it?, B. Seismol. Soc. Am., 84, 19401959, 1994.

Yoshida, S. and Kato, N.: Episodic aseismic slip in a two-degreeof-freedom block-spring model, Geophys. Res. Lett., 30, 16811684, 2003. 